\title{
Neuer Ansatz für die Second-Line-Therapie beim $\mathrm{mCRC}$
}

- Der Anti-VEGF-Antikörper Bevacizumab
(Avastin ${ }^{\oplus}$ ) kann das Gesamtüberleben der
Patienten nicht nur in der Erstlinie bis zur
Progression verlängern, sondern auch dann,
wenn die Therapie bei Tumorprogredienz in
der Zweitlinie fortgesetzt wird. Dass die
Wirksamkeit von Bevacizumab über mehre-
re Therapie-Linien erhalten bleibt, belegen
die Ergebnisse der TML (Treatment through
Multiple Lines)-Studie [Bennouna J et al.
Lancet Oncol. 2013;14(1):29-37]. An der
prospektiven randomisierten Phase-III-
Studie nahmen insgesamt 820 Patienten mit
mCRC teil. Als First-Line-Therapie hatten sie
Bevacizumab erhalten, kombiniert mit einer
Oxaliplatin- oder Irinotecan-basierten Chemotherapie. Nach Krankheitsprogression erhielten sie randomisiert eine erneute Bevacizumab-kombinierte Chemotherapie oder eine alleinige Chemotherapie. Die in der Erstlinie mit Oxaliplatin behandelten Patienten erhielten in der Zweitlinie ein Irinotecan-basiertes Regime und umgekehrt. Die in der Zweitlinie fortgeführte Gabe des Angiogenesehemmers war mit einer signifikanten Verbesserung des Gesamtüberlebens um 1,4 Monate assoziiert. In der Bevacizumab-Gruppe betrug das mediane Gesamtüberleben 11,2 Monate, in der allein mit Chemotherapie behandelten Gruppe
9,8 Monate (Hazard Ratio [HR] 0,81; $\mathrm{p}=$ 0,0062 ). Das progressionsfreie Überleben (PFS) wurde von 4,1 Monate unter alleiniger Chemotherapie auf 5,7 Monate verlängert (HR 0,68; $<<0,0001$ ). Die Tumorkontrollrate betrug in der Bevacizumab-Gruppe $68 \%$ gegenüber $54 \%$ in der Vergleichsgruppe ohne den Anti-VEGF-Antikörper ( $p<0,0001)$. Wie Stefan Kubicka, Reutlingen, herausstellte, wurde Bevacizumab auch bei fortgesetzter Gabe gut vertragen. Die Ergebnisse der TML-Studie eröffnen somit einen neuen Ansatz für die Secondline-Therapie bei Patienten mit $\mathrm{mCRC}$.

Abdol A. Ameri

Pressekonferenz "Avastin ${ }^{\circledR}$ beim metastasierten Kolorektalkarzinom ( $\mathrm{mCRC}$ ) - Therapie über die erste Tumorprogression hinaus", 17.01.2013, Berlin; Veranstalter: Roche

\section{Neue Therapieoption beim mehrfach vorbehandelten B-Zell-Lymphom}

— Die Prognose von Patienten mit mehrfach rezidiviertem oder therapierefraktärem aggressivem B-Zell-Lymphom (Non-Hodgkin-Lymphom, NHL) ist äußerst schlecht: „In der Regel beträgt die Lebenserwartung nur noch Wochen bis wenige Monate", erläuterte Norbert Schmitz, Hamburg. Mit dem neuartigen Zytostatikum Pixantron (Pixuvri甲) aus der Gruppe der Anthracenedione ist erstmals ein explizit für diese Indikation zugelassenes Präparat mit einer verbesserten Wirksamkeit gegenüber den bisher eingesetzten Zytostatika verfügbar, so der Hämatoonkologe.

Die Zulassung von Pixantron für die Monotherapie des mehrfach rezidivierten oder therapierefraktären aggressiven B-ZellLymphoms bei Erwachsenen erfolgte auf der Grundlage der Ergebnisse aus der offenen Phase-III-Multizenterstudie EXTEND (Expanding the reach of anthracyclines with piXanTronE in relapsed or refractory aggressive NHL Disease, [Pettengell R et al. Lancet Oncol. 2012;13(7):696-706]). Die insgesamt 140 Studienteilnehmer wurden randomisiert entweder mit sechs Zyklen Pixantron behandelt $\left(85 \mathrm{mg} / \mathrm{m}^{2}\right.$ intravenös an den Tagen 1, 8 und 15 eines 28-tägigen Zyklus) oder mit einem anderen, vom Untersucher ausgewählten Zytostatikum (Vinorelbin, Oxaliplatin, Ifosfamid, Etoposid, Mitoxantron oder Gemcitabin). „Bis zum Ende der Therapie sprachen $20 \%$ der Pati- enten in der Intention-to-Treat-Population auf Pixantron an, im Vergleichsarm nur 5,7\%", berichtete Schmitz.

Dieser signifikante Vorteil im Therapieansprechen $(p=0,021)$ blieb bis zum Ende der 18-monatigen Nachbeobachtungszeit erhalten. Mit Pixantron behandelte Patienten zeigten außerdem ein um $40 \%$ längeres progressionsfreies Überleben (5,3 vs. 2,6 Monate; $p=0,005)$ und ein im Trend längeres medianes Gesamtüberleben (10,2 vs. 7,6 Monate; $p=0,251$ ).
„Mit Pixantron steht eine neue Behandlungsmöglichkeit zur Verfügung, die viel weniger kardiotoxisch als die klassischen Anthrazykline ist", fügte Schmitz ergänzend hinzu, ,"das bringt die Palliativtherapie des mehrfach rezidivierten aggressiven BZell-Lymphoms einen großen Schritt voran". Silke Wedekind

Media-Roundtable: „Pixuvri ${ }^{\oplus}$ - Behandlung aggressiver B-Zell Lymphome jenseits der Erstund Zweitlinientherapie", Berlin, 18.01.2013; Veranstalter: CTI Life Sciences

\section{Unter die Haut}

— Bortezomib (Velcade ${ }^{\circledR}$ ) ist subkutan verabreicht zur Behandlung des multiplen Myeloms (MM) vergleichbar wirksam, aber besser verträglich als bei intravenöser Gabe. Erneut bestätigt dies die Nachbeobachtung der Patienten mit rezidiviertem oder refraktärem MM aus der Nicht-Unterlegenheitsstudie, die zur Zulassung der subkutanen Form geführt hatte [Arnulf B et al. Haematologica. 2012;97(12):1925-8]. Nach im Median über 17 Monaten lag das Gesamtansprechen in beiden Gruppen bei $52 \%$, eine Komplett- oder annähernd komplette Remis- sion erreichten $23 \%$ der Patienten bei Subkutangabe von Bortezomib und $22 \%$ bei intravenöser Gabe. Die Rate der peripheren Neuropathien war in der Subkutangruppe auch nach dem längeren Beobachtungszeitraum weiterhin deutlich niedriger als bei intravenöser Applikation (38 vs. $53 \%$ ), wobei auch Neuropathien des Grads II und III deutlich seltener waren. Dabei verbesserten sich die Neuropathien bei Subkutangabe von Bortezomib häufiger und rascher. red

Nach Informationen von Janssen-Cilag 